REGARDS

SUR LEECONOMIE ALLEMAND

BULLETIN ECONOMIQUE DUCIRAC
Regards sur l'économie allemande

Bulletin économique du CIRAC

$68 \mid 2004$

Varia

\title{
Le Brandebourg en quête d'une nouvelle image
}

\section{Marie-Hélène Pautrat}

\section{OpenEdition}

\section{Journals}

Édition électronique

URL : http://journals.openedition.org/rea/3626

DOI : $10.4000 /$ rea.3626

ISBN : 978-2-8218-0832-4

ISSN : 1965-0787

Éditeur

CIRAC

Édition imprimée

Date de publication : 1 octobre 2004

Pagination : 27-32

ISSN : 1156-8992

Référence électronique

Marie-Hélène Pautrat, "Le Brandebourg en quête d'une nouvelle image », Regards sur l'économie allemande [En ligne], 68 | octobre 2004, mis en ligne le 05 novembre 2009, consulté le 14 novembre 2019. URL : http://journals.openedition.org/rea/3626 ; DOI : 10.4000/rea.3626 


\section{Le Brandebourg en quête d'une nouvelle image}

\section{Marie-Hélène Pautrat}

Land de tradition agricole et industrielle, le Brandebourg, recréé dans ses frontières actuelles le 3 octobre 1990, présente, quinze ans après la chute du Mur, une situation contrastée. Chômage de masse, désindustrialisation, désenchantement et départ des jeunes les plus qualifiés, mais aussi très forte hétérogénéité entre les régions du Land rendent compte de la complexité d'une situation économique et sociale précaire qui perdure. Alors que le processus de rattrapage stagne, les autorités entendent aujourd'hui afficher le visage d'une région résolument tournée vers l'avenir. A l'heure de l'élargissement, le Brandebourg espère tirer profit de sa situation géographique au centre de l'Europe des 25 et affirme haut et fort, contre toute fatalité, ses atouts en matière d'implantation économique. C'est dans ce contexte que les autorités tentent d'enclencher une dynamique de croissance s'appuyant à la fois sur des noyaux de croissance et des infrastructures scientifiques et d'innovation prometteuses, en particulier dans les sciences de la vie.

Le Brandebourg est le $5^{\mathrm{e}}$ Land allemand en termes de superficie $\left(29476 \mathrm{~km}^{2}\right)$. Situé au Nord-Est de l'Allemagne, il dispose d'une frontière commune de $252 \mathrm{~km}$ avec la Pologne. Sa particularité est qu'il entoure la capitale fédérale et se trouve de ce fait privé de centre géographique effectif. Land peu peuplé avec 2,593 millions d'habitants, soit 3,6\% de la population de la RFA, le Brandebourg se caractérise également par une très faible densité de population : elle est de $88 \mathrm{hab} / \mathrm{km}^{2}$ seulement contre 230 en moyenne allemande (Berlin : 3799 $\mathrm{hab} / \mathrm{km}^{2}$ ). La population est nettement plus concentrée dans les principales villes dont Potsdam (131 414 hab), Cottbus (103 847), Francfort sur Oder (68 351) ou Brandebourg sur la Havel (75 276), et à la périphérie de Berlin. Le Brandebourg est le seul Land oriental à avoir vu sa population augmenter légèrement dans l'ensemble depuis 1991 : mais il s'agit pour l'essentiel des conséquences de l'étalement urbain autour de Berlin (suburbanisation) et de la pression migratoire sur les zones de banlieue. Depuis 2001, le mouvement s'inverse d'ailleurs, de sorte que le Brandebourg pourrait perdre $7 \%$ de sa population d'ici 2020 du fait du vieillissement démographique.

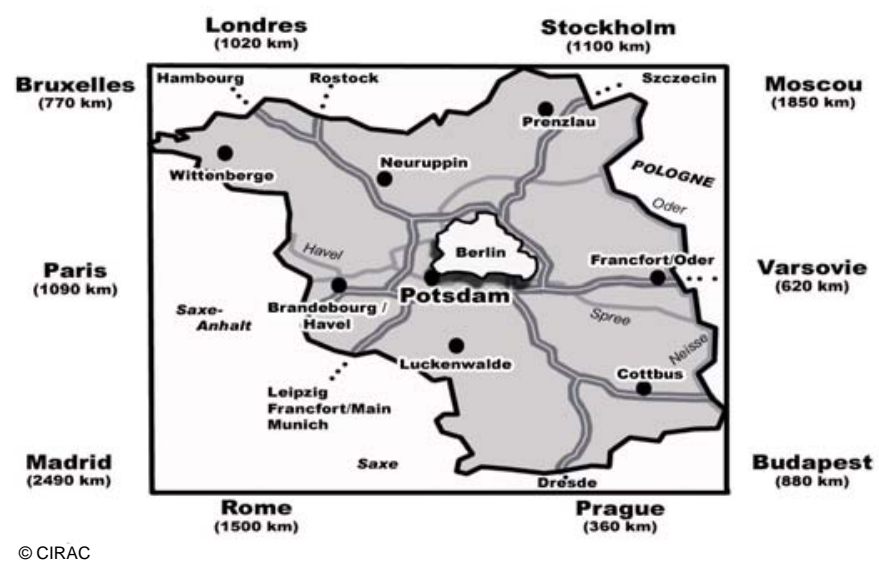

Un Land privé de centre géographique 
Brandebourg/Berlin, une région-métropole...

... à la recherche d'un équilibre territorial

Une dynamique de croissance grippée

Des indicateurs contrastés

Une situation sociale dégradée
Dès lors, il est aujourd'hui difficile de penser le Land de Brandebourg sans y associer Berlin, Land et capitale fédérale. Dans les faits, la séparation politique a été entérinée avec l'échec de la fusion à l'issue du référendum de 1996. Une seconde consultation pourrait néanmoins intervenir d'ici 2009. Les deux Länder forment, il est vrai, une entité de 6 millions d'habitants à l'échelle européenne. L'essor du Brandebourg semble plus que jamais passer par de nouvelles synergies avec Berlin qui, en exploitant les complémentarités des deux Länder, peuvent leur permettre d'accéder conjointement au rang de région majeure en Europe. C'est le sens de la coopération renforcée, concrétisée à partir d'avril 1995 par la signature d'une convention inter-étatique et déclinée depuis par différents outils : elle leur permet de planifier et de réaliser en commun l'aménagement durable de leur territoire.

Les deux Länder se présentent désormais ensemble dans toutes leurs actions de promotion internationale comme un espace économique et de vie commun sous l'étiquette de Hauptstadtregion Berlin/Brandenburg (« région-capitale »). Mais la région Berlin/Brandebourg n'en présente pas moins de très fortes disparités: en 2000, les trois quarts des six millions d'habitants résidaient dans la zone de concentration urbaine de Berlin et sa zone d'influence élargie. L'objectif principal de la politique de concertation en œuvre vise dès lors à promouvoir un développement polycentrique équilibré et structurant entre la capitale, sa proche banlieue, et le Brandebourg périphérique, tâchant d'éviter l'essor anarchique d'une banlieue hypertrophiée sur le modèle d'autres régions-métropoles.

Avec un PIB de 45,0 milliards $€$ en 2003, l'économie brandebourgeoise pèse toujours peu $(2,1 \%)$ dans le PIB allemand (2 112 milliards $€$ ) : elle arrive en $12^{\mathrm{e}}$ position, ex aequo avec la Saxe-Anhalt. Alors que sa dynamique de croissance est restée supérieure entre 1995 et 2001 à la moyenne des Länder de l'Ouest à faible croissance, la machine s'est grippée en 2002 dans le triple contexte d'une conjoncture déprimée, de transformations structurelles inachevées dans le bâtiment et l'artisanat, et des difficultés du commerce de détail. Cette situation accentue la stagnation du phénomène de convergence observée depuis 1997. Doublée d'un ralentissement des recettes fiscales, elle pèse sur la situation financière du Land qui peine à maintenir ses dépenses d'infrastructures.

En prix courants, le PIB par habitant atteignait $17476 €$ en 2003, légèrement en deçà de la moyenne des nouveaux Länder (moyenne RFA, Berlin inclus: $27431 €$ ). Mais dans le même temps, le PIB par actif s'élevait à $41529 €$ en 2003 (prix de 1995), devant les autres Länder orientaux. La productivité atteint en moyenne $79 \%$ du niveau national. Le retard continue donc de se combler très progressivement, et inégalement selon les secteurs d'activité et les entreprises : la productivité atteint par exemple $86 \%$ du niveau des anciens Länder dans le secteur minier ou l'industrie manufacturière, mais dépasse le niveau ouest-allemand pour la production de biens intermédiaires (104\%), et s'avère largement supérieure, dans certaines entreprises récentes, à celle d'entreprises comparables à l'Ouest (jusqu'à $120 \%$; source : ZAB).

Le marché du travail demeure certainement le principal défi pour la coalition SPD/CDU au pouvoir, comme le rappelle le score du parti néocommuniste PDS, qui a capitalisé le 19 septembre dernier (élection du Landtag) les suffrages des chômeurs et d'un électorat socialement fragilisé : le Brandebourg est l'un des Länder où les manifestations à l'encontre de la réforme des indemnités chômage (« Hartz IV », voir dans ce numéro) ont le plus mobilisé. En 2003 en effet, 253028 chômeurs y ont été enregistrés. Le taux de chômage se maintient à un niveau très élevé, proche de $17 \%$ chez les moins de 25 ans et supérieur à $18 \%$ pour l'ensemble de la population depuis 1997. Il dépasse largement la barre des $20 \%$ dans plusieurs districts (Uckermark : 25,5\% ; OberspreewaldLausitz : 24,3\% ; Frankfurt/Oder : 20,1\%), entretenu par la poursuite du recul de l'activité dans le secteur du bâtiment. Dans le même temps, le nombre d'actifs décroît de manière quasi continue depuis 1996 pour atteindre 1,008 million 
en 2003 : il se situe aujourd'hui au-dessous du 1,270 million enregistré après l'unification (1991). Ce mauvais score est d'ailleurs atténué par le nombre élevé d'habitants du Brandebourg (145 000 migrants alternants) qui occupent un emploi à Berlin. En dépit de salaires toujours inférieurs au niveau ouest-allemand, d'une très faible adhésion des entreprises brandebourgeoises aux accords de branche et d'un marché du travail flexible dans les faits, le problème est donc toujours la capacité de créer de l'emploi nouveau et de réduire le déficit structurel d'emplois réguliers.

Treize ans après l'unification, le Brandebourg, terre des anciens grands domaines fonciers, continue de présenter un caractère agricole, en dépit de profondes restructurations. Aujourd'hui, avec 4,2\% des actifs occupés, le secteur agricole $(2,5 \%$ du $\mathrm{PIB})$ pèse pourtant proportionnellement davantage que dans les autres Länder (moyenne RFA : 1,1\%), exception faite du Mecklembourg (4,3\%). On y compte 6709 exploitations (2003), dont près de la moitié dispose de moins de 20 ha (elles cultivent moins de $2 \%$ des surfaces agricoles), et environ 700 entre 500 et 2500 ha (60\% des surfaces agricoles), avec des niveaux de productivité souvent supérieurs à la moyenne allemande.

Mais le Brandebourg peut se prévaloir également d'une tradition industrielle confortée du temps de la RDA. Or avec les régions rurales, ce sont précisément les anciens fiefs et secteurs industriels traditionnels (construction automobile, chimie, construction ferroviaire et aéronautique) qui ont été le plus frappés au cours des années 90 par les restructurations, encore partiellement inachevées. Aujourd'hui, la base industrielle du Brandebourg (sans le bâtiment, secteur encore hypertrophié), certes assainie et stabilisée, demeure inférieure en termes d'emplois et de valeur ajoutée brute à celle des Länder occidentaux, alors que le secteur des services est comparable. Le profil industriel du Brandebourg est désormais marqué par quelques secteurs prépondérants comme la construction automobile (DaimlerChrysler à Ludwigsfelde et Berlin), l'industrie alimentaire (10 000 salariés, 157 entreprises ; CA : 2,5 milliards € en 2002), l'industrie des métaux (Brandebourg/Havel, Hennigsdorf ou encore Eisenhüttenstadt, créée de toutes pièces dans les années 1960 autour du complexe sidérurgique alimentant l'industrie de la RDA), ou l'industrie chimique. Avec 5000 salariés et un CA de 1,2 milliard $€$ en 2002, la chimie est concentrée autour de quelques pôles principaux (Schwedt, Schwarzheide, également Premnitz, Oranienburg ou Guben) et portée par quelques entreprises phares dont BASF Schwarzheide $\mathrm{GmbH}$, Altana Pharma Oranienburg $\mathrm{GmbH}$ ou Polyamid 2000 AG. Autour de ces secteurs se développent des réseaux de sous-traitance et de services.

Structure industrielle du Land de Brandebourg

\begin{tabular}{|lcc|}
\hline Principales branches & Chiffre d'affaires (en mio $€$ ) & Part de la branche \\
Industrie alimentaire & 2482,3 & $15 \%$ \\
Textile/habillement & 66,7 & - \\
Bois & 726,4 & $5 \%$ \\
Papier/Imprimerie & 1116,7 & $7 \%$ \\
Chimie/Matières plastiques & 1966,5 & $12 \%$ \\
Verre/Céramique & 1012,8 & $6 \%$ \\
Métal & 2180,1 & $14 \%$ \\
Construction mécanique & 1812,5 & $11 \%$ \\
Construction automobile & 3377,8 & $22 \%$ \\
Autre & 1296,6 & $8 \%$ \\
\hline
\end{tabular}

Source : Landesbetrieb für Datenverarbeitung und Statistik - Land Brandenburg 2003

Mais au regard d'un bilan économique très contrasté quinze ans après la chute du Mur, le Brandebourg s'interroge à son tour sur les moyens d'impulser une nouvelle dynamique à son économie : la discussion a fait rage ces dernières années sur l'utilisation optimale des aides, entre tenants d'une concentration des efforts sur les régions périphériques et rurales les plus nécessiteuses, ou d'une réorientation sur la ceinture porteuse de croissance autour de Berlin.
Une double tradition agricole...

... et industrielle

Le choix d'un renforcement des noyaux de croissance 
Quelques clusters émergents

Des conditions nouvelles avec l'élargissement européen

La perspective d'une stimulation des échanges extérieurs
Dans le même temps, le Land entend tirer les conséquences des effets de la désindustrialisation. Le développement du secteur des services (commerce, transports, information, services financiers, tourisme), qui occupe dorénavant plus des 2/3 des actifs du Land, n'ayant pas eu le résultat escompté en termes de création d'emplois et de croissance, le débat actuel sur un renforcement du tissu industriel porte sur une concentration des aides sur quelques « noyaux de croissance » (dans l'esprit du $2^{\mathrm{e}}$ Pacte de solidarité 2005-2019) : la constitution de clusters pourrait permettre de renforcer le potentiel économique des petites et moyennes entreprises. C'est cette stratégie de promotion de réseaux régionaux ou sectoriels, de constitution de pôles à fort potentiel de croissance et d'innovation, d'aides à la création et à l'implantation d'entreprises que poursuivent actuellement les autorités brandebourgeoises. Elles ont créé à cet effet en 2001 une agence de développement économique, la ZukunftsAgentur Brandenburg, SARL chargée à la fois de la promotion du Land auprès des investisseurs et du conseil aux entreprises locales (aides financières, conseil en matière d'innovation, de transfert de technologies, de marketing international, etc.). Elles espèrent de cette politique une consolidation du tissu industriel et, à terme, la revitalisation, par effet d'entraînement, d'autres pans de l'économie.

Quelques branches au fort potentiel d'innovation et de croissance organisent déjà leurs activités autour de réseaux où coopèrent entreprises innovantes et organismes de recherche : microtechnologies et microélectronique à Francfort/ Oder et au Sud de Berlin; aéronautique essentiellement autour de Berlin et Potsdam, optique. La constitution de clusters industriels concerne aussi certaines activités traditionnelles, comme la filière du bois ou encore le secteur de la logistique dont l'activité se développe aux alentours de la capitale et le long de la frontière germano-polonaise. Il s'agit de tirer profit de la position géographique du Brandebourg, centre de trafic et pont vers l'Europe orientale : infrastructures de transport - ferroviaires, routières, fluviales - rénovées et liaisons internationales avec notamment l'axe autoroutier Est-Ouest (A2), projet de concentration du trafic aérien sur le futur aéroport Berlin Brandeburg International. Quant à la capitale Potsdam, elle a est devenue un centre réputé de l'industrie cinématographique et audiovisuelle, autour du site historique des studios de Babelsberg où s'est constitué un important pôle d'activité : école supérieure du cinéma et de la télévision, High Tech Center, studios d'enregistrement, entreprises performantes (production de DVD, films en 3D).

Mais l'avenir de l'économie brandebourgeoise dépend aujourd'hui fortement de l'élargissement de l'Europe, avec ses atouts et ses contraintes. Situé aux confins de l'UE 15 et des zones économiques les plus actives, le Brandebourg bénéficie aujourd'hui du déplacement du centre de gravité européen. II est vrai qu'une partie des effets de l'intégration s'est progressivement fait sentir dès avant la date du $1^{\text {er }}$ mai 2004. Le Brandebourg s'est préparé 10 années durant à cette échéance par une intensification de la coopération transfrontalière avec les voïvodies polonaises de Lubusz et de Poméranie occidentale : actions dans le cadre de l'initiative communautaire Interreg (soutien aux régions frontalières), développement concerté de la région de l'Oder, programmes bilatéraux dans les domaines de la formation, de la lutte contre la criminalité, de l'environnement, instauration d'un dialogue entre partenaires sociaux, contacts entre partenaires économiques, coopération institutionnelle, programme du ministère de l'économie pour favoriser les investissements, etc.

L'élargissement apporte néanmoins des perspectives nouvelles en matière d'échanges extérieurs, possible moteur de croissance pour le Land. Selon l'institut de recherche DIW, le potentiel d'exportation de l'Allemagne orientale vers les nouveaux Etats membres demeure considérable. Ainsi, alors qu'en 2003 les exportations des entreprises brandebourgeoises s'élevaient à 5,07 milliards $€$ ( $+10,5 \%$ en un an), les seuls échanges vers l'Europe centrale et orientale se montaient à quelques 885 millions $€(+14,3 \%)$. Les Länder est-allemands peuvent en effet faire valoir leur connaissance particulière des pays de l'Est. 
Dans le même temps, les nouveaux membres bénéficieront des fonds structurels et de cohésion qui soutiendront leur économie (voisine du Brandebourg, la Pologne recevra 12,5 milliards $€$ entre 2004 et 2006). Mais la faible densité industrielle, la prépondérance d'entreprises de petite taille en proie à des difficultés de financement, le manque de personnel qualifié, notamment en raison du différentiel des salaires, et des investissements notoirement insuffisants en R\&D sont autant de facteurs qui pénalisent sur les marchés extérieurs les entreprises est-allemandes par rapport à leurs homologues d'Allemagne occidentale. Et l'atout de la proximité géographique avec les nouveaux marchés d'Europe centrale, particulièrement pertinent pour le Brandebourg, risque de pâtir du ralentissement du développement des liaisons routières et ferroviaires, du ressort de communes et Länder sous contrainte budgétaire.

Pourtant, et en dépit des mesures transitoires (notamment la limitation de la libre circulation des travailleurs), l'élargissement inquiète : le taux de participation aux dernières élections européennes s'est ainsi révélé extrêmement bas dans le Brandebourg (23\%). L'ouverture à l'Est renforce les craintes sur l'attractivité du Land, mise à mal ces dernières années par une série d'enquêtes comparatives sur la compétitivité des Länder (voir, par exemple, l'étude Cap Gemini). Dans un contexte de sous-emploi, c'est la question des coûts salariaux, avantage compétitif des nouveaux Etats membres, qui prédomine. Elle trouve par ailleurs son prolongement dans le débat actuel sur une zone à bas niveau de salaire à l'Est, que récusent les autorités du Land qui refusent de s'engager dans une concurrence avec les pays d'Europe centrale et orientale sur l'emploi et les coûts salariaux. Une récente étude de l'agence ZAB s'emploie donc à établir un inventaire des atouts du Brandebourg dans l'Europe élargie : qualité d'infrastructures modernes et rénovées, surfaces d'activités disponibles, programmes multiples d'aide aux investisseurs (aides à l'innovation, à la formation, mesures fiscales, crédits à taux privilégié), proximité d'un grand marché (Berlin) et potentiellement d'un marché élargi avec les nouveaux membres de l'UE. C'est aussi le sens de la campagne engagée au printemps par les autorités du Land pour améliorer l'image du site. Mais le Brandebourg peut surtout compter sur le niveau de qualification et de formation élevé de sa main d'œuvre et d'un environnement scientifique de grande qualité.

Le Brandebourg compte en effet 37500 étudiants (2003) dans ses 3 universités (université Viadrina de Francfort sur Oder, universités de Cottbus et de Potsdam) et 5 Fachhochschulen (établissements techniques supérieurs). Avec 19000 étudiants et 4000 emplois scientifiques, Potsdam figure en tête des villes allemandes pour la part de l'emploi scientifique dans la population. Mais à une plus large échelle, ces établissements viennent gonfler le vivier des 140000 étudiants berlinois : ils constituent ensemble un dispositif de formation d'une main d'œuvre hautement qualifiée, même si, il est vrai, les établissements subissent acutellement des restrictions de financement drastiques de la part de leur tutelle respective, et notamment du Land de Berlin. A cette offre de formation s'ajoute le potentiel scientifique : 21 centres de technologie, des centres de recherche réputés comme l'institut de recherche sur le climat (Potsdam), le centre de recherche sur les géosciences (Potsdam) ou le centre de recherche sur les membranes (Teltow). La recherche publique y joue un rôle essentiel dans le cas particulier des nouveaux Länder où il s'agit de pallier l'insuffisance de l'activité de R\&D d'entreprises souvent trop petites.

Dans ce contexte, le secteur porteur des sciences de la vie fait l'objet de toutes les attentions. Ici aussi, c'est l'offre régionale qui importe, et la capacité de synergies et de complémentarité avec la capitale Berlin. L'atout primordial de la région capitale est qu'elle bénéficie d'un tissu scientifique très dense (70 organismes de recherche extra-universitaire), d'hôpitaux universitaires renommés (la Charité à Berlin est le plus grand CHU d'Europe) et de 7 parcs biotechnologiques. Trois d'entre eux se situent dans le Brandebourg qui peut faire valoir, par rapport à Berlin, l'atout de surfaces disponibles répondant aux normes d'im-
Une volonté d'affirmer les atouts du Land

Un environnement scientifique exceptionnel

Une offre de qualité pour les sciences de la vie 
Premier site pour les biotechnologies en Allemagne plantation des laboratoires de pointe, à des coûts comparativement moins élevés. Les entreprises peuvent en outre bénéficier des aides européennes (Objectifs 1 et 2 pour 2000-2006) et du Bund à l'investissement.

Cette densité unique renforce la capacité de la région à constituer des réseaux entre organismes de recherche, partenaires économiques, universités et entreprises. La Biotechnologie-Region Berlin-Brandenburg (3 200 emplois), issue de la participation commune des deux Länder au concours fédéral Bio-Regio (1996), s'impose aujourd'hui comme le principal pôle allemand de R\&D dans les biotechnologies, notamment pour la biomédecine, les techniques médicales, la bioinformatique et les biotechnologies des plantes. Le secteur des sciences de la vie y compte une cinquantaine d'entreprises (pour l'essentiel autour de Berlin), plus d'une centaine dans la capitale, et plusieurs groupes pharmaceutiques: Schering, BASF, Altana, Sanofi-Synthelabo Menarini, etc. Au total, un quart des entreprises allemandes de biotechnologie est implanté dans la région, et les investissements progressent massivement depuis 1996, selon la ZAB : investissements dans les entreprises et investissements publics des deux Länder, du Bund (programmes InnoRegio pour soutenir l'innovation dans les nouveaux Länder, PRO INNO, concours EXIST-Transfer) et de l'Union européenne sous la forme de soutien à la constitution de réseaux et à la conduite de projets. Une agence commune aux deux Länder (BioTOP BerlinBrandebourg) coordonne en outre les activités de biotechnologie et de valorisation de l'offre régionale. Reste maintenant au Land à conforter le potentiel de croissance de la branche pour répondre à l'objectif fixé de devenir un des principaux clusters européens et mondiaux dans les sciences de la vie

APRES LA PHASE DOULOUREUSE DES RESTRUCTURATIONS et des ajustements, partiellement inachevée, les autorités brandebourgeoises s'appliquent à transmettre l'image d'une région tournée vers l'avenir. Si le Brandebourg n'avait jusqu'à présent pas été aidé par sa localisation géographique à la périphérie de l'Europe, la situation devrait changer et les autorités s'emploient à vanter ses atouts : atouts d'une économie de transition aux niveaux de salaires, de prix et de loyers modérés, qui présente dans le même temps des infrastructures modernes et un haut niveau de qualification. Et le Land peut faire valoir des synergies avec la capitale Berlin. Malgré cela, le tissu industriel reste insuffisamment dense pour garantir une croissance durable. Partant de ce constat, les pouvoirs publics s'emploient à concentrer leurs efforts sur quelques pôles d'activité à fort potentiel. Mais la dynamisation du Brandebourg passe également par la valorisation du potentiel scientifique et le passage à une économie basée sur la connaissance et l'innovation. C'est ainsi que le Brandebourg espère rompre la spirale vicieuse qui plane sur les Länder orientaux et créer sur place les conditions d'emploi d'une main d'œuvre qualifiée.

\section{Indications bibliographiques}

CAP Gemini, ERnst \& Young, Studie: Standortattraktivität. Die Unternehmerfreundlichkeit der Bundesländer im Vergleich. Kuzfassung, septembre 2002

« Brandenburgs Handel mit Polen: Struktur und Potentiale », DIW-Wochenbericht 33/2003

LANDESREgIERUNG BRANDENBURG, Jahreswirtschaftsbericht 2004 - Bilanz und Perspektiven der Brandenburger Wirtschaft, Ministerium für Wirtschaft des Landes Brandenburg, Potsdam, août 2004

Ministerium DER Finanzen Des LANDES BRAndenbuRgs, Forschrittsbericht „Aufbau Ost“ des Landes Brandenburg für das Jahr 2002, septembre 2003

Pautrat M.-H., «Pologne-RFA: les relations s'intensifient », Regards sur l'économie allemande, $\mathrm{n}^{\circ} 62$, juillet 2003

ZUKUNFTSAGENTUR BRANDENBURG, Brandenburg: Ein attraktiver Standort für ausländische Unternehmen - insbesondere bei höherwertigen Investitionen, mai 2004 (www.invest-in-brandenburg.com et www.zab-brandenburg.de)

«Sonderwirtschaftszone oder Förderung von Wachstumskernen - wie sollte eine zukünftige Ost-Förderung gestaltet werden? », ifo-Schnelldienst 10/2004 